\title{
De la ancianidad al adulto posmayor
}

\author{
ALEJANDRO KLEIN
}

Este trabajo busca ser una contribución para comprender algunos procesos de cambio por los que pasan los adultos mayores y la modificación del concepto de envejecimiento. Se revisan los conceptos de muerte desplazada, herencia y deuda, el lugar de la palabra sagrada y el de la confrontación transgeneracional de estos adultos mayores con sus propios antecesores. Por último, se indaga si es posible articular estos procesos con el concepto de "cuidado de si", de Foucault, que critica las ideas de Erikson respecto a la sabiduría como logro de esta etapa.

PALABRAS CLAVE: envejecimiento, herencia, confrontación, adultos mayores, palabra sagrada

\section{From Old Age to the Postmodern Adults}

This work aims to contribute to understand some of the change processes older adults live today and the transformation of the concept of aging. We review the concepts of displaced death, inheritance and debt, the place of the sacred word and the transgenerational confrontation of these older adults with their own grandparents. Finally, we examine whether it is possible to articulate these processes with Foucault's concept of "caring for", criticizing the ideas of Erikson regarding the wisdom as an achievement of this stage.

KEYWORDS: aging, heredity, confrontation, old age, sacred word 


\section{Introducción}

\section{$\mathrm{H}$}

asta hace unas décadas, la construcción de subjetividad tenía un límite claro: la vejez. Llegado a cierto punto, la jubilación indicaba el fin de cualquier promesa y el comienzo del fin, es decir, la vejez no indicaba sino el signo impostergable de la muerte como fin de cualquier anticipación, sin considerar posibles opciones religiosas. Sin embargo, las cosas han cambiado. Ahora la vejez no anticipa la muerte, sino una renovación de la promesa, con otro tipo de oportunidades, perspectivas y desafíos. No toda la población vieja participa de este novedoso clima cultural. Investigaciones más detalladas deberían determinar dónde se encuentran los límites sociales, culturales y económicos entre los viejos "tradicionales" y los de tipo más "rupturista".

\section{Antecedentes}

Se puede situar entre el siglo XVIII y el transcurso del XIX la fijación del estereotipo del anciano en sus aspectos clásicos: debilidad, precariedad y enfermedad, aspectos que se relacionan con la imposición del término "senil" (Katz, 1996). En especial, el cuerpo del anciano aparece en un proceso de déficit y pérdida permanente que se convierte en crónico e irreversible (Katz, 2000). Envejecer fue definido como una enfermedad progresiva, responsable de una variedad de cambios fisiológicos y anatómicos (Haber, 1986). En este sentido, se hablaba de "degeneración progresiva" como si fuera un estado normal y esperable (Katz, 1996).

De esta manera, el anciano era un ser improductivo e inútil socialmente. La observación de Iacub (2006) es relevante: se concebían dos muertes, para la sociedad y la muerte individual. El sujeto se iba apartando cada vez más de la vida y la sociedad para aislarse en su propio mundo (Cumming y Henry, 1961). También se podría analizar cómo estos procesos se asocian a factores de disciplinamiento y 
control cultural (Cole, 1997). Se puede señalar una historia cultural del envejecimiento que indica que el concepto mismo es difuso y muy cambiante según circunstancias históricas y contextos culturales. Esto implica que lo que se ha caracterizado como “científico" en el tema se ha revelado prejuicioso y paradigmático (Bourdelais, 1993).

Desde el siglo Xx, la concepción degenerativa y senil comienza a modificarse al mismo tiempo que se replantea desde la crítica el significado de las “edades" y la construcción de la biografía personal (Meyrowitz, 1984). La edad se torna un factor descriptivo irrelevante (Iacub, 2006) o se considera que entramos en una sociedad uniage en la que las fronteras entre edades se difuminan y tienden a unificarse (Neugarten, 1999). Al mismo tiempo, se alienta una revisión crítica de la noción de envejecimiento desde la segunda mitad del siglo xx (Butler, 1969). Se pone el acento en la continuidad más que en la discontinuidad; en la resiliencia y el potencial, más que en la pérdida y el déficit, y en las potencias y posibilidades que el envejecimiento podría implicar (Rosow, 1963; Neugarten, 1964; Atchley, 1977). Se plantean nuevas formas de inserción social (Ekerdt, 1986) y lo que el anciano puede aportar a la sociedad desde el concepto de "envejecimiento exitoso” (Baltes, Dittmann-Kohli y Dixon, 1984).

Para Baltes, el envejecimiento exitoso depende del esfuerzo aplicado a dominios en los que se mantiene el potencial de desarrollo. Por medio de este esfuerzo se logra una optimización de la funcionalidad, que compensa las pérdidas normativas y no normativas ocasionadas por el envejecimiento social y biológico. Por otro lado, para Rowe y Kahn (1997), la posibilidad de envejecimiento exitoso se relaciona con dos tipos de actividad: el mantenimiento de relaciones interpersonales satisfactorias y el mantenimiento de actividades productivas. Por lo tanto, lo que lo autores llaman compromiso "activo" con la vida es un factor relevante junto al adecuado funcionamiento físico y cognitivo, es decir, la capacidad de mantener un factor de autonomía y autocuidado, entre otros, y la baja probabilidad de padecer enfermedades crónicas y los riesgos asociados a ellas. En definitiva, entendemos que los autores proponen un modelo de vejez en el cual el adulto mayor puede enfrentar y resolver de manera autónoma sus problemas y estar inserto en actividades cotidianas. Para estos autores, los componentes mencionados están relacionados y se retroalimentan entre sí.

Por ejemplo, es posible constatar que la ausencia de enfermedad o discapacidad contribuye a mantener las funciones físicas y mentales necesarias para facilitar una participación activa en la vida social. Es interesante indicar que, al igual que Baltes, Dittmann-Kohli y Dixon (1984), se enfatiza en este artículo la necesidad de participación o compromiso con la vida como un factor esencial para un replanteamiento del sentido de la vida en los adultos mayores contemporáneos. Entendemos por “compromiso con la vida" la posibilidad de estructurar proyectos de vida satisfactorios que incluyan relaciones interpersonales, alta autoestima e inserción social. Podría indicarse que el concepto de envejecimiento exitoso se está enriqueciendo. Ya no se considera sólo la ausencia de enfermedades, sino la reformulación de la inserción del adulto mayor en la vida social y la reconfiguración de su biografía personal en términos de realizaciones y porvenir.

\section{Revisión empírica: la revolución gerontológica desde los aportes de la demografía y las ciencias sociales}

El Instituto Nacional de Estadística y Geografía (INEGI, 2010) registró en México un total de 10055379 adultos mayores, de los cuales 53\% son mujeres y $47 \%$ hombres. De acuerdo con la tasa de crecimiento anual en México, entre 1990 y 2010 el número de adultos mayores pasó de 5 a 10.1 millones, es decir que el incremento porcentual respecto 


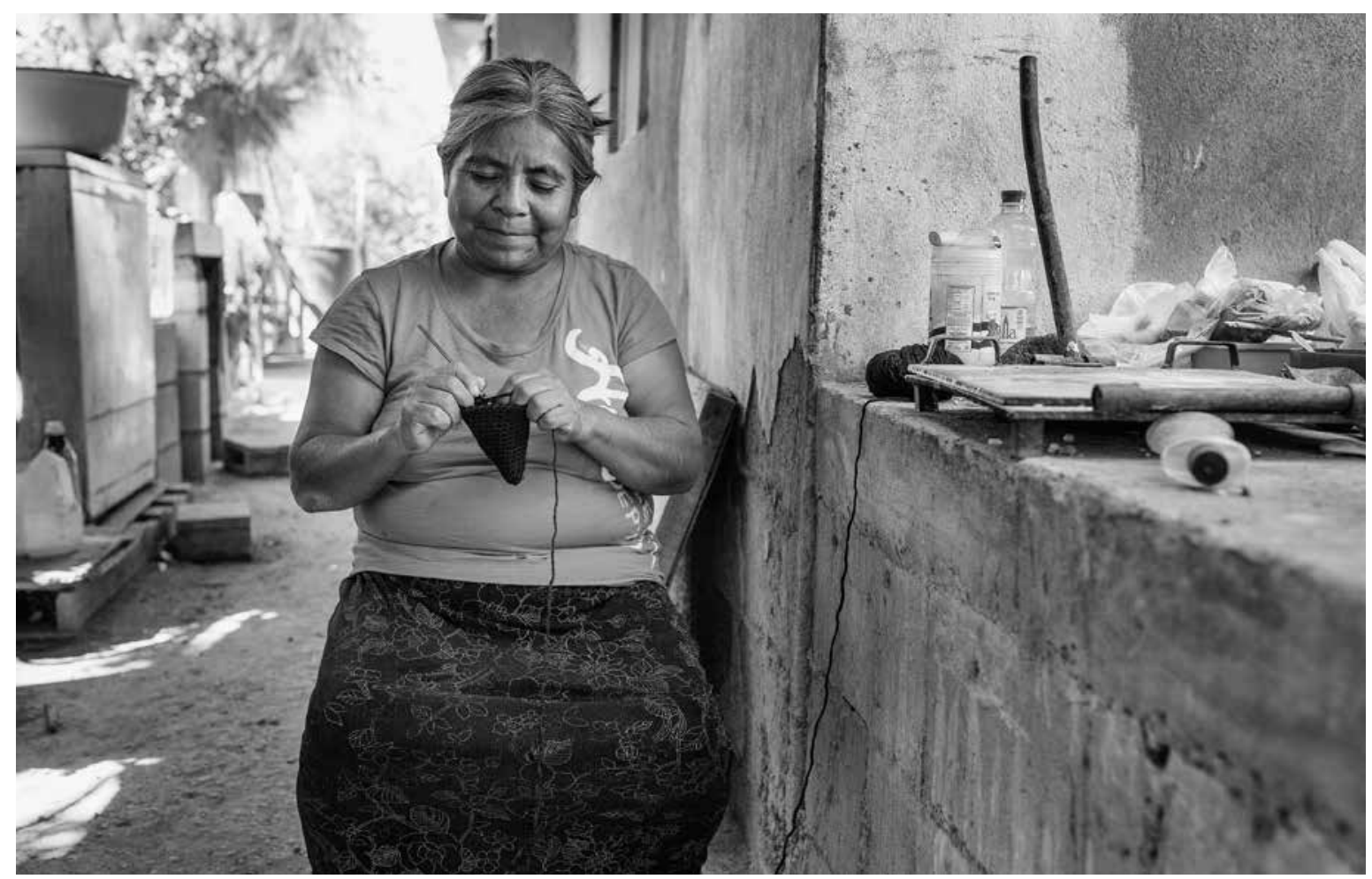

Prometeo Lucero • Mujer jornalera triqui en San Quintín. Baja California, México, 4 de octubre de 2014.

al total de la población fue de 6.2 a 9 (INEGI, 2011). Estos datos indican con claridad que México ha entrado en lo que se denomina envejecimiento poblacional, conjugado con la disminución de la tasa de natalidad (INEGI, 2005). Esto implica nuevos procesos de transición demográfica que, de acuerdo con los especialistas en población, conducirán a una tercera etapa de transición demográfica (Leeson y Harper, 2006; Dyson, 2010) dominada por una revolución gerontológica, por la cual se entiende que el siglo XXI es de los “centenarios” (Leeson, 2013).

Con base en las estimaciones realizadas por el Consejo Nacional de Población (Conapo, 2004), de 2010 en adelante "las personas de 65 años vivirán alrededor de 15 años más, expectativa que mostrará una tendencia a aumentar todavía más en los años venideros, manteniéndose cada vez más una etapa de sobre-mortalidad en dicho periodo de la vida"
(Villagómez, 2009: 307). Por otro lado, a partir del porcentaje de adultos mayores en la totalidad de la población, la Comisión Económica para América Latina y el Caribe (CEPAL, 2009) calculó que en 2010 la proporción de personas con 60 años o más en la región fue de $9.9 \%$, y será de 13\% en 2020 y de $25.8 \%$ en 2050. En Latinoamérica, la población de adultos mayores fue de 590 millones de personas en 2010 y la cifra se ha incrementado en los años siguientes (Leeson, 2013). Por otra parte, no sólo hay más adultos mayores, sino que viven cada vez más sin que haya, por el momento, un límite al alargamiento del curso de la vida (Leeson, 2009). A nuestro entender, esto se puede conceptualizar como el pasaje a una muerte desplazada indefinidamente, hecho inédito y crucial.

Es necesario indicar que el nivel de envejecimiento que presentan México y la mayoría de 
los países de la región se ha alcanzado en medio siglo. En Europa, el mismo proceso tomó dos siglos (Ham, 2003; Viveros, 2001; Camarano, 2004). Latinoamérica y el Caribe están próximos a enfrentar enormes desafíos en el área del envejecimiento global, junto con la necesidad de incluir el tema en las agendas de gobierno (Brea, 2003). La proporción de personas de 60 años será de $22 \%$ de la población total para 2050 (UN, 2010).

Por lo general, estos datos se consideran alarmantes. En este trabajo no se comparte ese punto de vista. Tenemos en cuenta la crisis de la seguridad social, la inseguridad económica y el aumento de enfermedades mentales y físicas; no obstante, otros datos indican la cualidad cambiante del mundo cambiante y lo imprevisible de algunos elementos de la estructura social. La revolución gerontológica está produciendo desafíos y oportunidades para gobiernos y ciudadanos en el mundo entero (Leeson y Harper, 2006; 2007a; 2007b; 2007c; 2008):

El desafío del incremento de la longevidad ha sido durante mucho tiempo asunto de interés demográfico, el cual no ha disminuido en años recientes. La escala del envejecimiento en el planeta es realmente inmensa y seguramente se considera una historia de éxito de la humanidad, puesto que cada vez más gente vive vidas largas y relativamente saludables (Leeson, 2013: 53). ${ }^{1}$

Otros autores más optimistas señalan un cambio en la estructura del trabajo y en la economía que implicará un dividendo demográfico (Lee y Mason, 2010) y estiman que existirán los recursos necesarios para asistir a la población envejecida (Heller, 2006). Desde una perspectiva social, hay datos que señalan aspectos positivos e integrativos culturalmente para los adultos mayores, como la posibilidad de resignificación del yo (Castro, 2001), la contribución cultural de los adultos mayores a las nuevas generaciones (Bosi, 1994), el incremento de la voluntad de vivir y de replantear historias y tradiciones culturales (Moragas, 1991), nuevas formas de aprendizaje (López La Vera, 2013), la reformulación y la valorización de la vida en pareja (Kemp y Kemp, 2002), de la sexualidad, el amor y la satisfacción marital (Bachand y Caron, 2001; Parker, 2000; Kaslow y Robinson, 1996; Noller y Fitzpatrick, 1993; Nina, 2004), y por último, el factor de generatividad, entendido como la capacidad de enfrentar retos y fortalecerse de manera subjetiva (Erikson, 1950).

En resumen, los adultos mayores hoy no sólo son más longevos, sino que disfrutan de mejor calidad y estilos de vida (Villar, 2013). Pensamos que es posible relacionar las nuevas formas de autopercepción y construcción de identidad con la llamada teoría de la desvinculación o desengache y ruptura. Ésta considera que el adulto mayor se aparta de la sociedad para centrarse más en su vida interior, lo que lo independiza de obligaciones sociales e incrementa su capacidad de satisfacción (Havighurst, Neugarten y Tobin, citados en Carstensen, 1990). Sin embargo, cuando situemos a los adultos mayores como "garantes" de la herencia social, quizá sea más apropiado hablar de "cambio" que de "apartamiento" en el vínculo con la sociedad.

Estas perspectivas señalan la necesidad de cambiar la imagen de los adultos mayores como una carga, para destacar su valor como recurso social y cultural, que se complementa con el dato fundamental de que cuando la persona mayor tiene los recursos necesarios depende menos de su familia, que pasa a segundo plano, lo que resulta en la ampliación de

1

The challenge of increasing longevity has long been an issue of demographic interest, and no less so in more recent years The scale of ageing across the globe is truly immense and must surely be regarded as one of the success stories of humankind as more and more people live long, relatively healthy lives (Leeson, 2013: 53). [La traducción es mía.] 
redes sociales y de amistad, y fortalece el sentido de vitalidad (López La Vera, 2010; 2013; Pick et al., 2007; Sánchez, 2002).

Otros autores prefieren tomar estos datos como reveladores de empoderamiento y agencia, basados en investigaciones que registran el deseo de los adultos mayores de tomar el control de sus vidas (Soria, 2006), incrementar el sentido del yo y la confianza en sí mismos (Sánchez, 2002), así como la capacidad de enfrentar nuevos aprendizajes y desafíos (López La Vera, 2013). Guzmán, Huenchuan y Montes de Oca (2003) plantean que el cumplimiento de una vejez activa requiere que el Estado asuma un papel activo en la mejora de las condiciones de vida de los adultos mayores.

Discrepamos con esta perspectiva: los adultos mayores han modificado sus posibilidades de vida con, sin o contra el Estado. Es posible que esto se relacione con que las redes sociales sustituyen aquello de lo que aquél carece (Uchino, Cacioppo y Kiecolt-Glaser, 1996; Mendes de León et al., 1999). Se ha comprobado que poseer una red social adecuada tiene innumerables efectos positivos en el área de la salud física y mental, mejora la autopercepción que los adultos mayores tienen de sí mismos y acrecienta el sentido de satisfacción vital (Irvine et al., 1999; Gaete, Rivera y Román, 2009; Muchinik, 1984). Así, los fenómenos que describimos en relación con el desplazamiento de la muerte se respaldan en numerosas investigaciones demográficas, antropológicas, psicológicas y sociológicas, de autores norteamericanos, europeos y latinoamericanos, que describen aspectos inherentes a la sociedad del envejecimiento y la revolución gerontológica.

\section{Paradigmas ambiguos}

Todos estos cambios implican la aparición de lo que en otro trabajo llamé paradigmas ambiguos (Klein, 2010a), es decir, la situación de cómo referirse a algo que recibía una denominación tradicional y consensuada que ya no puede utilizarse, lo que implica que cualquier denominación no ha de ser sino acotada, injusta o generadora de malestar. En el caso del envejecimiento, cualquier denominación que usemos será incómoda. ¿Se trata de "viejos"? Sí y no. ¿Son "adultos mayores”? Sí y no. ¿Hablamos de la "tercera edad”? Sí y no. Estos malentendidos conceptuales son también ambigüedades conceptuales. No indican, sino que plantean nuevas modalidades culturales - que algunos calificarán de posmodernas-y de construcción de subjetividad, punto que retomaré al final de este trabajo. De manera provisional, llamaremos a esta nueva estructura psicosocial "viejos-no viejos" o "posadultos".

Estimamos que el escándalo actual del grupo "rupturista" de los viejos radica en que ya no aceptan ser viejos. No aceptan el mandato generacional de la decrepitud, por así decirlo. En ese punto, hacen una verdadera confrontación transgeneracional con resultados imprevisibles. Se ha hablado de una revolución feminista, y en ese sentido bien se podría hablar de una revolución gerontológica; una, protagonista de comienzos del siglo Xx, la otra, protagonista del XXI; las dos con antecedentes y prolegómenos que es necesario desmenuzar. La novedad o el "escándalo" no se sitúa sólo en la gimnasia, en las dietas, en la práctica sexual renovada - hasta hace poco un tema tabú-, en las búsquedas emocionales o en la concreción de proyectos educativos alternativos dentro o fuera de las llamadas universidades de la tercera edad. Coincidimos en que todos estos factores se coadyuvan entre sí en la abolición de otro tabú aún más significativo: la muerte. Los viejos ven delante suyo una segunda, tercera o cuarta oportunidad en términos de proyectos, es decir, ven "vida" y no "muerte", unida a un fortalecimiento de las estéticas corporales no decrépitas dentro de una renovación portentosa del “cuidado de sí” foucaultiano (Foucault, 1988). 


\section{Los cambios familiares y generacionales y los nuevos posicionamientos del adulto mayor}

Según datos demográficos, es posible indicar que el progresivo aumento del envejecimiento poblacional no sólo acrecentará el número de personas mayores en el total de la población - en el caso mexicano, se estima que en 2050 será 28\% (Conapo, 2004)_, sino que implica cambios sustanciales en la composición de las familias (Mancinas y Garay, 2013). En síntesis, la familia contemporánea atraviesa profundas transformaciones en relación con la estructura de la denominada familia nuclear, las técnicas reproductivas, la resignificación de la identidad sexual, y en especial, el replanteamiento del lugar social y familiar de la mujer (Vasconcelos y Morgado, 2005; Negreiros y Féres-Carneiro, 2004). Aparecen nuevas estructuras familiares en las sociedades industrializadas representadas por diferentes modalidades vinculares (Harper, 2003). Es posible destacar como una de sus características un descenso del índice de fertilidad por el uso extendido de contraceptivos, pero también por un aplazamiento de la maternidad por oportunidades en el mercado laboral que la mujer no poseía antes (Harper, 2003; Hoff, 2007; Wainerman, 1996).

En el caso de México, Tuirán (1995) indica que según la Encuesta Nacional de Actitudes y Valores de 1994, la familia se relaciona con emociones positivas y acogedoras, que generan expectativas y demandas. Cabe indicar que este universo refleja un imaginario social y no necesariamente una realidad fáctica. Montes de Oca (2004) indica que la expectativa que genera la familia no implica su capacidad para resolver problemas sociales u otros.

Las familias multigeneracionales suman un porcentaje significativo en el territorio mexicano (Rodríguez, 2010; Montes de Oca, 2004; 2009), con varios procesos de cohabitación (Hakkert y Guzmán, 2004). Las investigaciones señalan una reducción del tamaño de los hogares, disminución de la presencia relativa de los hogares nucleares y aumento de los hogares no nucleares, aumento en la proporción de hogares con jefatura femenina y "envejecimiento” de los hogares (López Ramírez, 2001). En 2006, se estimaba en 27.1\% el total de hogares "envejecidos” (Montes de Oca y Garay, 2010a). Se ha incrementado también el número de hogares unipersonales (Montes de Oca y Garay, 2010b). De esta manera, la prolongación de la expectativa de vida lleva a una mayor comunicación entre generaciones, que aparecen más valorizadas dentro de lo que se denomina proceso de verticalización de la familia (Abellán y Esparza, 2010; Bazo, 2008). Esto implica una reformulación de los intercambios financieros y emocionales entre padres e hijos (Bengtson, 2001) y entre abuelos y nietos (Klein, 2007; 2010b).

Es interesante reiterar que en las hipótesis de este trabajo los adultos mayores, si cuentan con las condiciones económicas adecuadas, prefieren vivir solos y preservar su autonomía (Mancinas y Garay, 2013). En algunos casos, los hijos dependen de los padres (Hakkert y Guzmán, 2004) y los abuelos cuidan de sus nietos (Saraceno, 2008; Klein, 2010b). Los investigadores señalan que el envejecimiento poblacional en general (Harper, 2004), y en particular en la población mexicana, ha tenido una influencia decisiva en las organizaciones familiares (Mancinas y Garay, 2013; OMS, 2002). Estos cambios se pueden enfocar desde la perspectiva del estudio de tradiciones intergeneracionales (Harper, 2004), que señalan el pasaje de familias multigeneracionales a la intersección de relaciones generacionales (Bengtson, 2001; Harper, 2003).

Por nuestra parte, advertimos que uno de los factores que inciden en estos cambios familiares es que los adultos mayores de hoy — no todos, pero muchos- no quieren ser viejos como en los modelos heredados (Klein, 2010a). No transmiten esos modelos porque no quieren reproducirlos (Klein, 2010a; 2010b). Hay un efecto de detención de la transmisión intergeneracional, probablemente 
inédita en las historias de las mentalidades y las culturas, con mayor simetría de los vínculos (Vidal y Menzinger, 2005; Hoff, 2007; Leeson, 2013).

Llamamos "confrontación transgeneracional" (Klein, 2013) a esta diferencia generacional y entendemos que es parte de la subjetividad de estos abuelos posadultos. Tomamos el término "confrontación" de Winnicott (1972), porque lo postula como una necesidad de marcar y establecer diferencias generacionales que adquiere su fuerza en la construcción de nuevas formas de subjetividad, lo que incide en un sentido de confianza y autovaloración en los sujetos que la llevan adelante (Bleichmar y De Bleichmar, 1999); esto, a su vez, los legitima desde una nueva posición social (Klein, 2007).

\section{De la palabra sagrada a la cuestión de lo transmisible}

Desde estas nuevas realidades sociales y subjetivas, la palabra del adulto mayor ya no parece sagrada, incuestionable, digna de ser escuchada:

A cada generación le toca recuperar y reelaborar el pasado con distintos instrumentos culturales, mismos que pone en juego en su esfuerzo por comprenderse a sí misma, a la generación que le precedió y a la generación que le sigue [...] el paso generacional responde, en buena medida, a los modos en los que cada generación ubica su memoria (Avendaño, 2010: 6).

Entonces, podemos pensar que la nuestra se caracteriza, antes que nada, por profundas discontinuidades sociales, culturales y económicas. Las operaciones de recibir, transformar y anticipar (Kaës, 1994) sufren modificaciones esenciales desde las cuales se cuestiona el significado de transmitir y de lo transmisible (Kaës, 1993; 1996; Puget y Käes, 1991). Si tomamos en cuenta que transmisión, memoria colectiva, memoria individual y consolidación de la vivencia temporal están íntimamente entrelazadas, cuando un elemento de esta matriz se debilita o desaparece, surgen patologías o reposicionamientos culturales en la transmisión generacional. Kaës (1996) se inclina a analizar los problemas de la modernidad en torno a las dificultades de transmisión (Beck, 1997; Enríquez, 1990).

Por ello, es importante destacar la idea de que la sociedad keynesiana es una sociedad de herederos. Para que haya heredero, debe existir herencia disponible y capacidad de aceptar la muerte, que marca la diferencia entre generaciones, por medio de la posibilidad de un duelo - en definitiva, un trabajo de la memoria-, que resignifica la historia generacional y subjetiva. Sugerimos que si ya no hay palabra sagrada no es porque ya no haya transmisores o herederos, sino porque existe una reformulación profunda de ese relato sagrado o herencia a transmitir, lo que va más allá de que algo necesariamente se tenga que transmitir. Esta idea, netamente freudiana (Freud, 1921), no descarta que aquello transmitido sea "nada", un "vacío", una "trampa", una "estafa" o una "deuda" desde nuevas condiciones de contrato social, que operan como "descontractualización generalizada” (Klein, 2006).

\section{Deudas que se transmiten como impagables por generaciones}

Esta situación genera una reestructuración general de la identidad, del problema de la herencia y lo heredable y de los vínculos desde nuevas formas sociales (Sader y Gentili, 1999). Implica la prevalencia del sentimiento de amenaza constante - " "sensación de catástrofe inminente" (Klein, 2006) - por el miedo crónico, por ejemplo, a perder el empleo (Araújo, 2002), el cual, después de la humillación, pasa a ser un hecho innegable tanto como su correlato, la necesidad del sometimiento (Forrester, 2000). De 


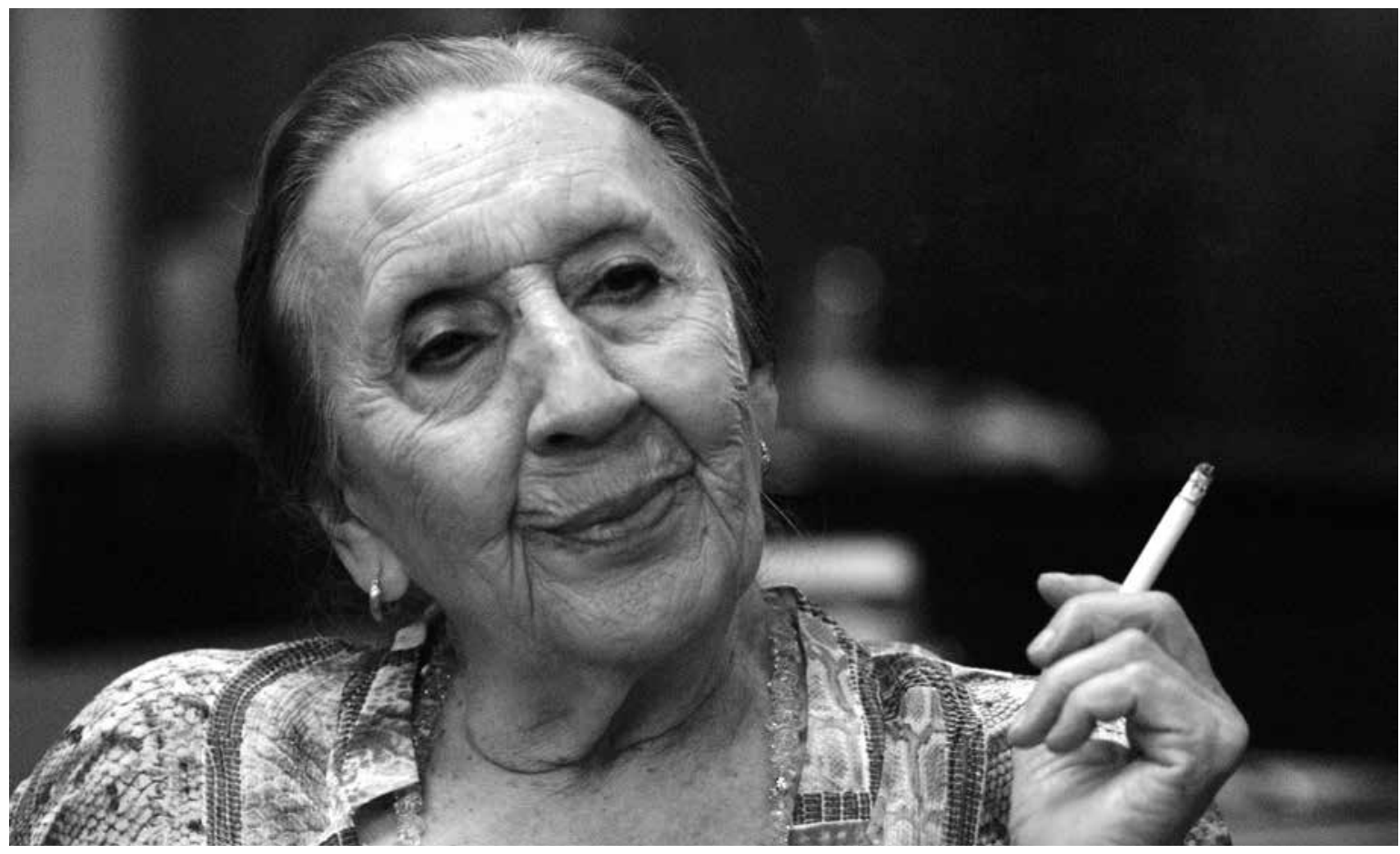

RICARDo RAmíREZ ARrIOLA/ARCHIVO360.com • Guillermina Bravo en el XXVII Festival Internacional de Danza Contemporánea Lila López. Bailarina, coreógrafa, fundadora y directora del Ballet Nacional y del Centro Nacional de Danza Contemporánea en Querétaro. San Luis Potosí, México, 13 de octubre de 2007.

manera progresiva, no hay nada que transmitir sino una deuda, que se torna impagable:

Hay una deuda que se paga para arriba, pero que, fundamentalmente, se paga para abajo. La deuda contraída con los padres, se paga con los hijos. Y esta es una deuda imperiosa, acuciante e impostergable. Es una deuda que no puede eludirse. Cuando las circunstancias externas nos impiden saldarla —deshonrados por no poder honrar nuestras obligaciones- [...] se nos impone como humillación insoportable [...]. Eso quiere decir que, por primera vez, una generación entera se ve impedida de pagar la deuda contraída [...] para que podamos asegurarles a nuestros hijos, lo mismo que nuestros padres nos dieron a nosotros (Volnovich, 2002: 1-2).

Esta deuda social, que se impone y se hace crónica, reconfigura el lugar de los herederos, la herencia y la memoria colectiva. Desde allí se plantea la hipótesis de una situación de amnesia criptográfica, por la cual se instala el olvido - o mejor, la indiferencia- en lugar de la memoria, lo desheredado en lugar de la herencia y lo expulsado precario en lugar de los herederos discriminados. Ya no es claro cómo y hasta qué punto se es parte de una continuidad generacional, lo que a su vez se enlaza con el problema de las formas de paranoia radicadas cada vez más en la cotidianidad (Klein, 2006; 2013).

\section{Los abuelos como garantes sin garantías}

El "adulto mayor, no adulto mayor" parece mantener entonces su posición de ser transmisor, pero ya sin herencia o con una herencia endeudante, que se hace por ello intransmisible. Son garantes, entonces, de un proceso que ya no tiene garantías. Garantizan 
de alguna manera una continuidad generacional aun desde la discontinuidad. Desde un proceso de ucronía, introducen algún tiempo de temporalización, que no es asimilable por completo al de memorización, que incluye en sí la noción de herencia. Desde allí se retoma la figura del garante para sustituir la figura de la herencia: estos posadultos mayores garantizan que al menos algo se puede hacer, aunque no siempre quede claro qué y cómo. Si no siempre se verifican procesos intergeneracionales, sí es posible indicar una distancia generacional que opera en términos de estructuración y ordenación simbólica.

\section{Conclusiones}

Hemos indicado cómo el proceso de envejecimiento se torna cada vez más parte de la construcción de identidad de un sujeto con características novedosas: el adulto posmayor, el que construye su subjetividad desde un fuerte campo de indagación y experimentación. El adulto posmayor encuentra y se construye nuevos contextos sociales y culturales que le permiten revalorizarse y cuidarse a sí mismo, y siente que es respetado en sus decisiones y puntos de vista. La visión tradicional del adulto mayor de Erikson (2000), como un ser que llega al final de la vida con un sentido de integración y plenitud, que acepta la vida que ha vivido y por ende la muerte que tiene por delante, se modifica cuando la muerte ya no es un orientador sólido y se ubica cada vez más en un lugar de desplazamiento progresivo.

Colocamos al adulto mayor en por lo menos cuatro dimensiones que se entrelazan: la de un intenso proceso de resignificación de su identidad, dentro de lo que distinguimos la confrontación transgeneracional; por consiguiente, su cuestionamiento de la vejez como signo de su identidad; la dificultad de encontrar una nominación adecuada para su definición dentro de lo que llamamos paradigmas ambiguos, y el debate sobre si éstos son o no transmisores de una herencia que parece transformarse rápidamente en deuda social. En este sentido, los procesos de transmisión generacional se están modificando de manera sustancial, lo que conlleva un replanteamiento del lugar social del adulto mayor.

Algunos especialistas hablan en específico de envejecimiento posmoderno para plantear esta novedad que queremos señalar en relación con el adulto mayor (Featherstone y Hepworth, 1991). Asimismo, aunque el tema no se ha tocado en el trabajo, es necesario mencionar que algunos autores (Neugarten, 1999; Iacub 2006) señalan la creciente irrelevancia de la edad como diferenciador de la identidad, sustituida por un proceso biográfico de tipo transetario, en el que las edades se mezclan o se tornan indiscernibles, ambiguas o innecesarias (Neugarten, 1999). Desde el punto de vista de las nuevas configuraciones familiares provenientes de la sociedad del envejecimiento, hemos indicado que son intersecciones generacionales, lo que parece generar vínculos tanto de solidaridad como de agobio y extenuación (Arriagada, 2007). La cuestión no está resuelta y es materia de discusión entre los especialistas.

Como indicamos, parece que los adultos mayores de hoy están decididos a vivir más y mejor que los adultos mayores de generaciones precedentes. De repente se ha vuelto crucial que el adulto mayor sea activo y productivo, en la medida de lo posible. El desplazamiento de la muerte inminente favorece estos procesos. Planteamos en este artículo cuestiones que no han sido debatidas apropiadamente debido a que ha predominado un sentido apocalíptico de la sociedad de envejecimiento, rebatido recientemente (Leeson, 2013). Al mismo tiempo, los procesos señalados parecen indicar que estamos frente a fuertes procesos de transición que auguran una variedad de tendencias gerontológicas y sociales, algunas francamente impredecibles. Este trabajo intenta ser una contribución al respecto. D 


\section{Bibliografía}

Abellán García, Antonio y Cecilia Esparza Catalán, 2010, “Solidaridad familiar y dependencia entre las personas mayores”, en Informes Portal Mayores, núm. 99.

Araújo, Ana María (coord.), 2002, Impactos del desempleo. Transformaciones en la subjetividad, Alternativas, Montevideo.

Arriagada, Irma, 2007, "Transformaciones familiares y políticas de bienestar en América Latina”, en Irma Arriagada (comp.), Familias y políticas públicas en América Latina. Una historia de desencuentros, Comisión Económica para América Latina y el Caribe/Fondo de Población de las Naciones Unidas (Libros de la CEPAL, 96), Santiago, pp. 125-152.

Atchley, Robert, 1977, The Social Forces in Later Life. An Introduction to Social Gerontology, Wadsworth, Belmont.

Avendaño Amador, César Roberto, 2010, "Memoria colectiva y memoria personal: apuntes para pensar una psicología política”, en Revista Electrónica de Psicología Iztacala, vol. 12, núm. 2, pp. 45-57.

Bachand, Leslie y Susan Caron, 2001, "Ties that Bind, a Qualitative Study of Happy Long-Term Marriages”, en Contemporary Family Therapy, vol. 23, núm. 1, pp. 105-121.

Baltes, Paul, Freya Dittmann-Kohli y Roger Dixon, 1984, "New Perspectives on the Development of Intelligence in Adulthood: Toward a DualProcess Conception and a Model of Selective Optimization with Compensation”, en Paul Baltes y Orville G. Brim, Jr. (coords.), LifeSpan Development and Behavior, vol. 6, Academic Press, Nueva York, pp. 33-76.

Bazo, María Teresa, 2008, "Personas mayores y solidaridad familiar", en Política y Sociedad, vol. 45, núm. 2, pp. 73-85.

Beck, Ulrich (coord.), 1997, Modernización reflexiva: política, tradición y estética en el orden social moderno, Alianza (Alianza Universidad), Madrid.

Bengtson, Vern L., 2001, "Beyond the Nuclear Family: The Increasing Importance of Multigenerational Relationships in American Society", en Journal of Marriage and the Family, vol. 63, núm. 1, pp. 1-16.

Berenstein, Isidoro y Janine Puget, 1988, Psicoanálisis de la pareja matrimonial, Paidós, Buenos Aires.

Bleichmar, Norberto y Celia de Bleichmar, 1999, El psicoanálisis después de Freud. Teoría clínica, Paidós, México.

Bosi, Ecléa, 1994, Memórias e sociedade, lembranças de velhos, T. A. Editor, São Paulo.

Bourdelais, Patrice, 1993, L'âge de la vieillesse. Histoire du vieillissement de la population, Odile Jacob, París.

Brea, Jorge, 2003, "Population Dynamics in Latin America”, en Population Bulletin, vol. 58, núm. 1, pp. 1-36.

Butler, Robert, 1969, "Age-Ism: Another Form of Bigotry”, en The Gerontologist, vol. 9, pp. 243-246.

Camarano, Ana Amélia, 2004, Os novos idosos brasileiros: muito além dos 60?, Instituto de Pesquisa Econômica Aplicada, Río de Janeiro.

Carstensen, Laura, 1990, "Cambios relacionados con la edad en la actividad social”, en Laura Carstensen y Barry A. Edelstein (eds.), Gerontología clínica. Intervención psicológica y social, Martínez Roca, Barcelona, pp. 58-73.

Castro, Odair, 2001, Envelhecer, um encontro inesperado?, Notadez Universidade Tuiuti do Paraná, Sapucaia do Sul.

Cole, Thomas, 1997, The Journey of Life, Cambridge University Press, Cambridge.

Comisión Económica para América Latina y el Caribe (CEPAL), 2009, "Políticas públicas y crisis de cuidado en América Latina: alternativas e iniciativas”, en Panorama Social de América Latina 2009, Comisión Económica para América Latina y el Caribe, Santiago, pp. 227-240.

Consejo Nacional de Población (Conapo), 2004, “El envejecimiento de la población en México”, disponible en línea: <http://www.conapo.gob. mx/es/CONAPO/Envejecimiento_de_la_poblacion_de_Mexico_reto_del_Siglo_XXI>. Consultado el 8 de febrero de 2012.

Cumming, Elaine y William Henry, 1961, Growing Old: The Process of Disengagement, Basic Books, Nueva York.

Dyson, Tim, 2010, Population and Development, Zed Books, Londres y Nueva York.

Ekerdt, David, 1986, "The Busy Ethic: Moral Continuity between Work and Retirement”, en The Gerontologist, vol. 26, pp. $239-244$.

Enríquez, Micheline, 1990, “La envoltura de memoria y sus huecos”, en Anzieu Didier (coord.), Las envolturas psíquicas, Amorrortu, Buenos Aires. Erikson, Erik, 1950, Childhood and Society, Norton, NuevaYork.

— 2000, El ciclo vital completado, Paidós, Madrid.

Featherstone, Mike y Mike Hepworth, 1991, "The Mask of Ageing and the Postmodern Life Course”, en Mike Featherstone, Mike Hepworth y Bryan S. Turner (eds.), The Body, Social Process and Cultural Theory, Sage, Thousand Oaks.

Forrester, Viviane, 2000, Una extraña dictadura, Fondo de Cultura Económica, Buenos Aires.

Foucault, Michel, 1988, "A tecnologia política dos individuos", en Michel Foucault, Ética, Sexualidade, Política, vol. V: Ditos \& Escritos, Forense Universitária, Río de Janeiro. 
Freud, Sigmund, 1921, Psicología de las masas y análisis del yo, t. XVIII, Amorrortu, Buenos Aires.

Gaete Fiscella, José Manuel, Jesús Rivera Navarro y Helena Román, 2009, "Aplicación del modelo de redes personales al estudio de los ancianos dependientes”, en Revista Multidisciplinar de Gerontología, vol. 19, núm. 3, pp. 121-129.

Guzmán, José Miguel, Sandra Huenchuan y Verónica Montes de Oca, 2003, "Redes de apoyo social de las personas mayores, marco conceptual”, en Notas de Población, año XXIX, núm. 77, pp. 35-70.

Haber, Carole, 1986, "Geriatrics: A Specialty in Search of Specialists", en David Van Tassel y Peter N. Stearns (eds.), Old Age in Bureaucratic Society: The Elderly, the Experts, and the State in American History, Greenwood Press, Westport, pp. 66-84.

Hakkert, Roberto y Julio Guzmán, 2004, "Envejecimiento demográfico y arreglos familiares de vida en América Latina”, en Marina Ariza y Orlandina de Oliveira (coords.), Imágenes de la familia en el cambio de sig/o, Universidad Nacional Autónoma de México-Instituto de Investigaciones Sociales, México.

Ham Chande, Roberto, 2003, El envejecimiento en México: el siguiente reto de la transición demográfica, Porrúa, México.

Harper, Sarah, 2003, "Changing Families as European Societies Age”, European Journal of Sociology, vol. 44, núm. 2, pp. $155-184$.

, 2004, Families in Ageing Societies, A Multi-Disciplinary Approach, Oxford University Press, Oxford.

Heller, Peter, 2006, Is Asia Prepared for an Ageing Population?, documento de trabajo WP/06/272, International Monetary Fund, Washington, D. C.

Hoff, Andreas, 2007, "Functional Solidarity between Grandparents and Grandchildren in Germany", documento de trabajo 307, Oxford Institute of Ageing, Oxford.

lacub, Ricardo, 2006, Erótica y vejez. Perspectivas de Occidente, Paidós, Buenos Aires.

Instituto Nacional de Estadística y Geografía (INEGI), 2005, Los adultos mayores en México. Perfil sociodemográfico al inicio del sig/o XXI, disponible en línea: <http://www.inegi.org.mx/prod_serv/contenidos/espanol/bvinegi/productos/censos/poblacion/adultosmayores/ Adultos_mayores_web2.pdf>. Consultado el 30 de agosto de 2013.

,2010, “Censo de Población y Vivienda 2010. Levantamiento censal. Manual del entrevistador del cuestionario ampliado”, disponible en línea: <http://www3.inegi.org.mx/sistemas/biblioteca/detalle.aspx?c=27626\&s=est\&upc=702825002061\&pf=Pob\&f=2\&cl=0\&tg=0>. Consultado el 5 de julio de 2014.

, 2011, Estadísticas a propósito del día Internacional de las Personas de Edad. Datos nacionales, disponible en línea: <http://www.inegi. org.mx/inegi/saladeprensa/aproposito/2014/adultos0.pdf>. Consultado el 12 de mayo de 2012.

Irvine, Jane et al., 1999, "Depression and Risk of Sudden Cardiac Death after Acute Myocardial Infarction, Testing for the Confounding Effects of Fatigue", en Psychosomatic Medicine, vol. 61, núm. 6, pp. 729-737.

Kaës, René, 1993, El grupo y el sujeto del grupo. Elementos para una teoría psicoanalítica del grupo, Amorrortu, Buenos Aires.

__ 1994, La dimensión psicoanalítica de grupo, Asociación Argentina de Psicología y Psicoterapia de Grupo, Buenos Aires. (coord.), 1996, Transmisión de la vida psíquica entre generaciones, Amorrortu, Buenos Aires.

Kaslow, Florence y James Robinson, 1996, "Long-Term Satisfying Marriages, Perceptions of Contributing Factors”, en The American Journal of Family Therapy, vol. 24, núm. 2, pp. 153-173.

Katz, Stephen, 1996, Disciplining Old Age. The Formation of Gerontological Knowledge, University Press of Virginia, Charlottesville.

__ 2000, "Busy Bodies: Activity, Aging, and the Management of Everyday Life”, en Journal of Aging Studies, vol. 14, núm. 2, pp. 135-152.

Kemp, Jerrold y Edith Kemp, 2002, Older Couples, New Romances, Finding and Keeping Love in Later Life, Celestial Arts, Berkeley.

Klein, Alejandro, 2006, Adolescentes sin adolescencia: reflexiones en torno a la construcción de subjetividad adolescente bajo el contexto neoliberal, Psicolibros-Universitario, Montevideo.

- 2007, Los padres y docentes tenemos derecho a saber. Algunas pistas para comprender mejor al mundo adolescente, PsicolibrosWaslala, Montevideo.

,2010a, "Neoliberalismo-Neoevangelismo-Cambios sociodemográficos. Posibles marcos epistemológicos frente a algunos desafíos actuales en el campo de las ciencias sociales (los paradigmas ambiguos)", en Acciones e Investigaciones Sociales, núm. 27, pp. 69-109.

2010b, "Nuevas formas de relacionamiento abuelos-nietos adolescentes desde los cambios demográficos-sociales actuales", en Psicología Revista, vol. 18, núm. 1, pp. 1-25.

—_, 2013, Subjetividad, familias y lazo social. Procesos psicosociales emergentes, Manantial, Buenos Aires.

Lee, Ronald y Andrew Mason, 2010, “Fertility, Human Capital, and Economic Growth over the Demographic Transition”, en European Journal Population, vol. 26, núm. 2, pp. 159-182.

Leeson, George, 2009, "Demography, Politics and Policy in Europe”, en Peter Ludlow (ed.), Setting EU Priorities 2009, European Strategy Forum, Ponte de Lima, pp. 102-124. 
_ 2013, "The demographics of population ageing in Latin America, the Caribbean and the Iberian Peninsula, 1950-2050", en Verónica Montes de Oca (coord.), Envejecimiento en América Latina y el Caribe: enfoques en investigación y docencia de la Red Latinoamericana de Investigación en Envejecimiento, Universidad Nacional Autónoma de México-Instituto de Investigaciones Sociales, México, pp. 53-71.

Leeson, George y Sarah Harper, 2006, "Attitudes to Ageing and Later Life”, en Global Ageing Survey, reporte de investigación 106, Oxford Institute of Ageing, Oxford.

__, 2007a, "Ageing and Later Life. United Kingdom and Europe”, en Global Ageing Survey, reporte de investigación 107, Oxford Institute of Ageing, Oxford.

—_, 2007b, "Ageing and Later Life. The Americas", en Global Ageing Survey, reporte de investigación 207, Oxford Institute of Ageing, Oxford. ,2007c, "Ageing and Later Life. Hong Kong and Asia", en Global Ageing Survey, reporte de investigación 307, Oxford Institute of Ageing, Oxford

—_, 2008, "Some Descriptive Findings from the Global Ageing Survey (GLAS), Investing in Later Life", en Global Ageing Survey, reporte de investigación 108, Oxford Institute of Ageing, Oxford.

López La Vera, Blanca, 2010, "La transición entre ocupar el tiempo libre y empoderar. Perspectivas de la educación universitaria dirigida al adulto mayor", en Ageing Horizons, núm. 9, pp. 82-99.

- 2013, "Empoderamiento y adultos mayores. Impacto de la participación de un grupo de adultos mayores en un programa educativo", en Verónica Montes de Oca (coord.), Envejecimiento en América Latina y el Caribe: enfoques en investigación y docencia de la Red Latinoamericana de Investigación en Envejecimiento, Universidad Nacional Autónoma de México-Instituto de Investigaciones Sociales, México, pp. 207-248.

López Ramírez, Adriana, 2001, El perfil sociodemográfico de los hogares en México 1976-1997, Consejo Nacional de Población, México.

Mancinas Espinoza, Sandra y Sagrario Garay Villegas, 2013, “Familia, envejecimiento y políticas sociales”, en Verónica Montes de Oca (coord.), Envejecimiento en América Latina y el Caribe: enfoques en investigación y docencia de la Red Latinoamericana de Investigación en Envejecimiento, Universidad Nacional Autónoma de México-Instituto de Investigaciones Sociales, México, pp. 395-424.

Mendes de León, Carlos et al., 1999, "Social Networks y Disability Transitions across Eight Intervals of Yearly Data in the New Haven EPESE", en Journal of Gerontology, Psychological Sciences-Social Sciences, vol. 54B, núm. 3, pp. S162-S172.

Meyrowitz, Joshua, 1984, No Sense of Place: The Impact of Electronic Media on Social Behaviour, Oxford University Press, Oxford.

Montes de Oca, Verónica, 2004, "Envejecimiento y protección familiar en México, límites y potencialidades del apoyo al interior del hogar", en Marina Ariza y Orlandina de Oliveira (coords.), Imágenes de la familia en el cambio de sig/o, Universidad Nacional Autónoma de México-Instituto de Investigaciones Sociales, México, pp. 510-563.

,2009, "Families and Intergenerational Solidarity in Mexico, Challenges and Opportunities", en Seminar on "Family Support Networks and Population Ageing 2009", disponible en línea: <http://www.unfpa.org/webdav/site/global/shared/documents/publications/2010/ family_support_networks2009.pdf>. Consultado el 12 de junio de 2013.

Montes de Oca, Verónica y Susana Garay, 2010a, "Familias, hogares y vejez, cambios y determinantes en los arreglos familiares con personas adultas mayores en México, 1992-2006”, en Ana María Chávez Galindo y Catherine Menkes Bancet (comps.), Procesos y tendencias poblacionales en el México contemporáneo. Una mirada desde la ENADID 2006, Centro Nacional de Equidad de Género y Salud Reproductiva/Secretaría de Salud, México.

__ 2010b, "Living Arrangements and Old People, Changes and Determinants of Older Persons in Regions on Mexico, 1992-2000", ponencia presentada en la Population Association of America 2010 Annual Meeting, Dallas, del 15 al 17 de abril.

Moragas, Ricardo, 1991, Gerontología social, envejecimiento y calidad de vida, Herder, Barcelona.

Muchinik, Eva, 1984, Hacia una nueva imagen de la vejez, Belgrano, Buenos Aires.

Negreiros, Teresa y Terezinha Féres-Carneiro, 2004, "Masculino e feminino na família contemporânea”, en Estudos e Pesquisas em Psicología, año 4, núm. 1, pp. 34-47.

Neugarten, Bernice, 1964, Personality in Middle and Late Life, Atherton Press, Nueva York. , 1999, Los significados de la edad, Herder, Barcelona.

Nina Estrella, Ruth, 2004, "Sabor a mí... Pareja y vejez", en Carmen Sánchez (ed.), Familia y vejez: memoria, Red de Estudios de la Vejez/ Proyecto Atlantea/Universidad de Puerto Rico, San Juan, pp. 117-130.

Noller, Patricia y Mary Ann Fitzpatrick, 1993, Communication in Family Relationships, Prentice Hall, Englewood Cliffs.

Organización Mundial de la Salud (oms), 2002, “Envejecimiento activo, un marco político”, en Revista Especializada de Geriatría y Gerontología, vol. 37, núm. S2, pp. 74-105. 
Parker, Robyn, 2000, “How Partners in Long-Term Relationships View Marriage”, en Family Matters, vol. 55, pp. 74-88.

Pick, Susan et al., 2007, “Escala para medir agencia personal y empoderamiento (ESAGE)", en Interamerican Journal of Psychology, vol. 41, núm. 3, pp. 295-304.

Puget, Janine y René Kaës, 1991, Violencia de estado y psicoanálisis, Centro Editor de América Latina, Buenos Aires.

Rodríguez, Katya, 2010, Poverty in Mexico at the Beginning of the Twenty First Century, An Alternative Analysis, Lambert Academic Publishing, Saarbrücken.

Rosow, Irving, 1963, Social Integration of the Aged, Free Press, Nueva York.

Rowe, John y Robert Kahn, 1997, "Successful Aging", The Gerontologist, vol. 37, núm. 4, pp. 433-440.

, 1998, Successful Aging, Pantheon, Nueva York.

Sader, Emilio y Pablo Gentili (coords.), 1999, La trama del neoliberalismo. Mercado, crisis y exclusión social, Editorial Universitaria de Buenos Aires, Buenos Aires.

Sánchez, Alfonso, 2002, "Dispositivos de empoderamiento para el desarrollo psicosocial”, en Universitas Psychologica, vol. 1, núm. 2, pp. 39-48.

Saraceno, Chiara, 2008, "Introduction, Intergenerational Relations in Families. A Micro-Macro Perspective”, en Chiara Saraceno (comp.), Families, Ageing and Social Policy. Intergenerational Solidarity in European Welfare States, Edward Elgar, Cheltenham, pp. 1-19.

Soria, Carlos, 2006, "Empoderamiento, un medio, no un fin", en Patricio Crespo (coord.), Empoderamiento, ¿tomar las riendas?, Asocam/ Plataforma Latinoamericana de Gestión de Conocimientos, Quito, pp. 45-51.

Tuirán, Rodolfo, 1995, "Cambios y arraigos tradicionales", en Demos, núm. 8, pp. 30-31, disponible en línea: <http://www.ejournal.unam.mx/ dms/no08/DMS00815.pdf>. Consultado el 1 de septiembre de 2014.

Uchino, Bert, John Cacioppo y Janice Kiecolt-Glaser, 1996, "The Relationship between Social Support y Physiological Processes. A Review with Emphasis on Underlying Mechanisms y Implications for Health”, en Psychological Bulletin, vol. 119, núm. 3, pp. 488-453.

United Nations (UN), 2010, World Population Prospects. The 2010 Revision, Nueva York.

Vasconcelos, Eduardo, 1988, "Estado y políticas sociales en el capitalismo: un abordaje marxista”, en Elisabete Borgiani y Carlos Montaño (coords.), La política social hoy, Cortez Editora (Biblioteca Latinoamericana de Servicio Social), São Paulo.

Vasconcelos, Eduardo y Rossana Morgado, 2005, Subsidios analíticos e metodológicos para a atuação no Sistema Único de Assistência Social (sUAS) e do Programa de Atendimento Integral à Familia, Programa de Atendimento Integral à Família/Secretaria de Atenção à Saúde, Río de Janeiro.

Vidal, Susana y Jorge Menzinger, 2005, "New Technologies in the Commnication between Grandparents and Grandchildren in Spain", en Generations Review, vol. 15, núm. 4, pp. 26-32.

Villagómez Ornelas, Paloma, 2009, El envejecimiento demográfico en México: niveles, tendencias y reflexiones en torno a la población de adultos mayores, Instituto Nacional de Geriatría, México.

Villar, Feliciano, 2013, "Desafíos de la investigación interdisciplinaria en gerontología”, en Verónica Montes de Oca (coord.), Envejecimiento en América Latina y el Caribe: enfoques en investigación y docencia de la Red Latinoamericana de Investigación en Envejecimiento, Universidad Nacional Autónoma de México-Instituto de Investigaciones Sociales, México, pp. 189-206.

Viveros Madariaga, Alberto, 2001, Envejecimiento y vejez en América Latina y el Caribe: políticas públicas y las acciones de la sociedad, Comisión Económica para América Latina y el Caribe/Centro Latinoamericano y Caribeño de Demografía/Fondo de Población de las Naciones Unidas (Población y Desarrollo, 22), Santiago.

Volnovich, Juan Carlos, 2002, “El default con nuestros hijos: la desesperación por no cobrar. El dolor de no poder pagar”, en Clarin.com, disponible en línea: <http://edant.clarin.com/diario/2002/01/30/o-01901.htm>. Consultado el 6 de abril de 2012.

Wainerman, Cristina (ed.), 1996, Vivir en familia, Organización de las Naciones Unidas para la Educación, la Ciencia y la Cultura/Losada, Buenos Aires.

Winnicott, Donald, 1972, Realidad y juego, Gedisa, Barcelona. 